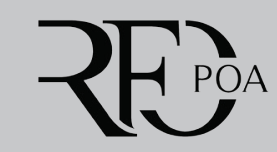

\title{
ODONTOLOGIA HOSPITALAR NO BRASIL: ONDE ESTAMOS? UMA ANÁLISE DO CENÁRIO DOS ÚLTIMOS ANOS
}

\author{
Hospital dentistry in Brazil: where are we? \\ An analysis of the scenario of the past few years
}

D Gabriela Elen Moreira Silvaa, (D) Luciana Pereira da Rocha Thomsena, (D) Júlio César Tanos de Lacerdab, D Sérgio Henrique Benfenatti Botelho ${ }^{b}$, (D) Júbila Aparecida Costa Reis ${ }^{b}$, (iD) Raí Douglas de Araújo Ferreirac, (ID Renata Gonçalves Resende ${ }^{a, b}$

\begin{abstract}
RESUMO
A Odontologia Hospitalar visa à manutenção e aos cuidados prestados a pacientes sob internação hospitalar. O cirurgião-dentista é um profissional devidamente capacitado para identificação, prevenção e tratamento de alterações na cavidade bucal. A inserção do CD em ambiente hospitalar tem sido instituída mediante resoluções e leis para beneficiar quem carece desse atendimento. Sendo assim, o objetivo deste trabalho é apresentar trajetória da Odontologia Hospitalar no Brasil, verificar seu panorama atual e mostrar sobre a importância desse profissional na equipe multidisciplinar dos hospitais. As buscas foram realizadas, entre os anos de 2001 e 2019, em bases de dados bibliográficos nacionais e como complemento no manual de odontologia hospitalar, em matérias publicadas pela Agência Nacional de Vigilância Sanitária e em leis publicadas nos diários oficiais dos municípios, estados e união. Os dados mostram que muitos estados já se adequaram, do ponto de vista legal, no sentido de tornar a Odontologia Hospitalar uma realidade. No entanto, ainda falta fiscalização e profissionais ocupando todos os postos de trabalho. Logo, percebe-se que a última década foi decisiva em prol da Odontologia Hospitalar, mas ainda há muito o que se fazer para que novos avanços aconteçam.
\end{abstract}

Palavras-chave: Saúde bucal. Unidades de terapia intensiva. Equipe de assistência ao paciente. Legislação.

\begin{abstract}
Hospital Dentistry aims at the maintenance and care provided to patients under hospitalization. The dental surgeon is a professional duly trained to identify, prevent and treat changes in the oral cavity. The insertion of the DS in a hospital environment has been instituted through resolutions and laws to benefit those who need this service. Therefore, the objective of this work is to present the trajectory of $\mathrm{OH}$ in Brazil, to verify its current panorama and to show about the importance of this professional in the multidisciplinary team of hospitals. The searches were carried out between 2001 and 2019 in national bibliographic databases and as a complement to the hospital dentistry manual, in articles published by the National Health Surveillance Agency and in laws published in the official diaries of the municipalities, states and union. The data show that many states have already adapted, from the legal point of view, to make hospital dentistry a reality. However, inspection and professionals are still lacking in all jobs. Therefore, the last decade has been decisive in favor of hospital dentistry, but there is still a lot to be done for new advances to happen.
\end{abstract}

Keywords: Oral health. Intensive care units. Patient care team. Legislation.

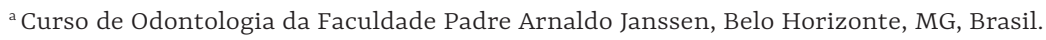

${ }^{\mathrm{b}}$ Hospital Metropolitano Odilon Behrens, Belo Horizonte, MG, Brasil.

${ }^{\mathrm{c}}$ Instituto Biocor, Nova Lima, MG, Brasil.
}

Autora de correspondência: Renata Gonçalves Resende - E-mail: renatagresende@yahoo.com.br

Data de envio: 19/01/2020 | Data de aceite: 03/02/2020 


\section{INTRODUÇÃO}

Define-se como Odontologia Hospitalar $(\mathrm{OH})$ os cuidados odontológicos prestados a pacientes internados em ambientes hospitalares ${ }^{1}$. A atuação do profissional cirurgião-dentista (CD) abrange prevenção, diagnóstico e tratamento de doenças da cavidade bucal. Por estar restrito ao leito e não poder se deslocar aos consultórios convencionais, e pela repercussão sistêmica dessas doenças, o paciente necessita desses cuidados em ambiente hospitalar².

A presença do CD nos hospitais faz-se necessária para se ter uma avaliação integral do indivíduo, redução de custos para as instituições de saúde através da identificação, prevenção e tratamento de alterações na cavidade bucal. Com isso, há uma diminuição do uso de insumos de altos custos, como nutrição parenteral, medicações e redução da taxa de ocupação de leitos ${ }^{3}$. O perfil dos pacientes que serão assistidos por esse profissional, na maior parte das vezes, é oncológico, em pós-operatório cirúrgico de cabeça e pescoço, transplantados, com necessidades especiais, infectados gravemente e pacientes submetidos a tratamento intensivo ${ }^{4}$. Se houver acompanhamento de saúde bucal desses pacientes, diversos comprometimentos sistêmicos poderão ser minimizados ou até mesmo evitados, como endocardite bacteriana, descompensação glicêmica, pneumonia nosocomial, entre outras patologias 5 .

A OH é uma modalidade de trabalho que permite ao CD a ampliação da sua área de atuação. No Brasil, a OH já existe há muitas décadas, representadas por profissionais isolados ou instituições, mas sua consolidação formal teve início após a criação da Associação Brasileira de Odontologia Hospitalar (ABRAOH) em 2004, além de diversas legislações e especificações sobre o exercício da odontologia em ambiente hospitalar. Embora existam tais estratégias que introduzem o dentista no ambiente hospitalar ainda há uma ausência de fiscalização por parte dos governantes ${ }^{6}$.

Diante disso, torna-se relevante apresentar a trajetória da $\mathrm{OH}$ no Brasil, verificar seu panorama atual e discorrer sobre a importância dessa especialidade na equipe multidisciplinar dos hospitais.

\section{MÉTodos}

Como base de dados foram utilizados a Biblioteca Virtual de Saúde (BVS) e Scientific Electronic Library Online (SCIELO), Literatura Latino-Americana e do Caribe em Ciências da Saúde (LILACS) e como complemento o Manual de Odontologia Hospitalar, matérias publicadas pela Agência Nacional de Vigilância Sanitária (ANVISA) e leis publicadas nos diários oficiais dos municípios, estados e união.

Os critérios de inclusão do estudo foram artigos publicados em eixo temporal de dezoito anos, entre 2001 e 2019, em idioma português. Os critérios de exclusão foram publicações que não apresentaram texto na íntegra, resumos que não eram pertinentes ao tema e trabalhos que não tinham o Brasil como assunto. Isso porque essa busca refere-se à realidade da $\mathrm{OH}$ no Brasil, sendo pertinente restringir a essa pesquisa a literatura nacional.

Para localizar as publicações indexadas nestas bases de dados, foram utilizados os seguintes descritores: saúde bucal, unidade de terapia intensiva, equipe de assistência ao paciente, legislação, intervenção, saúde bucal. 


\section{REVISÃO DE LITERATURA DISCUTIDA}

\section{História da Odontologia Hospitalar nas Unidades de Tratamentos Intensivos no Brasil}

As Unidades de Tratamentos Intensivos (UTIs) foram criadas no Brasil, em 1971, com o intuito de intensificar os cuidados a pacientes graves ${ }^{7}$. Com o passar do tempo, verificou-se a necessidade de ampliação dos cuidados especializados aos pacientes, surgindo assim a implementação de CD nesse ambiente ${ }^{4}$.

De acordo com Morais ${ }^{8}$ o atendimento de $\mathrm{OH}$ surgiu no final da década de 70 . Contudo, somente em 2000 foi criado o Serviço de Odontologia Hospitalar na Santa Casa de Misericórdia de Porto Alegre, voltado para o setor de cirurgias e traumatologias bucomaxilofaciais. Os serviços de tratamento intensivos com a introdução do CD começaram a funcionar no Brasil a partir de 2005, na Santa Casa de Misericórdia de Barretos ${ }^{6}$.

Por questões financeiras, a maior parte da população brasileira é assistida pelo Sistema Único de Saúde (SUS) para sanar as emergências odontológicas. São poucos os locais que fornecem este tipo de atendimento imediato. Devido à demora e o sucateamento desse serviço de saúde, há uma piora no quadro clínico desses pacientes podendo chegar à internação em UTIs ${ }^{9}$.

Segundo Paim ${ }^{10}$, há uma ausência de hierarquização e gestão do atendimento odontológico pelos municípios, bem como a descentralização do atendimento fornecido pelo SUS. Ademais, os princípios e doutrinas preconizadas pelo SUS em 1990, não vem sendo seguidas pelos municípios ${ }^{11}$.

Diante disso e devido às diversas complicações que a falta de cuidados bucais pode causar aos pacientes críticos, a presença de um profissional especializado nesses centros de internação, vem sendo cada vez mais solicitada.

\section{Importância da Odontologia Hospitalar}

As equipes multidisciplinares das UTIs são compostas por diversos profissionais com a finalidade de abranger os cuidados ao paciente como um todo, visto que as áreas da saúde estão interligadas. Cada profissional na sua área é capaz de fornecer informações e ações a fim de promover conforto e qualidade de vida ao paciente ${ }^{12}$.

O paciente internado em UTI está de cinco a dez vezes mais propenso a infecção, devido às condições sistêmicas, aos procedimentos invasivos e a deficiência imunológica do indivíduo ${ }^{13}$. Um dos mecanismos comumente utilizados para sobrevida desses pacientes é a respiração artificial, que gera como consequência a hipossalivação, deixando a cavidade bucal mais suscetível à colonização de diversas bactérias ${ }^{14}$. Estima-se que o paciente que necessita de respiração artificial possui de 7 a 21 vezes a chance de desenvolver pneumonia associada à ventilação mecânica (PAVM) ${ }^{15}$.

No entanto, Souza e colaboradores ${ }^{16}$ afirmam que com a implantação do bundle de prevenção da PAVM e o protocolo de higiene bucal reduz de 33,3\% para 3,5\% a incidência dessa doença. Compreende como bundle, medidas baseadas em evidências, a fim de prevenir a PAVM. Tais medidas são: elevação da cabeceira da cama entre $30^{\circ}$ e $45^{\circ}$; redução diária da sedação e avaliação de extubação; profilaxia de úlcera péptica e profilaxia de trombose venosa ${ }^{16}$.

O acúmulo do biofilme na cavidade bucal de pacientes sob internação hospitalar é fator etiológico para o desencadeamento de doenças infecciosas nas estruturas bucais como cárie, doenças periodontais, necrose pulpar, candidíase, dentre outras. Além disso, pode provocar um comprometimento sistêmico e influenciar na terapêutica medicamentosa ${ }^{17}$. 
Para Gomes e Esteves ${ }^{13}$, pacientes em tratamento intensivo possuem deficiência na higienização bucal devido ao tempo de internação e à complexidade do biofilme bucal, sendo este o foco de infecções hospitalares. Além do mais, Pereira e d'Ottaviano ${ }^{18}$, corroboram que a presença de um profissional qualificado para cuidar do sistema estomatognático contribui significativamente para a prevenção de doenças ororespiratórias que prejudicam a recuperação do paciente.

\section{Legislação Brasileira}

Em fevereiro de 2010, foi elaborado pela ANVISA a Resolução da Diretoria Colegiada no 7 (RDC-07), que determina as condições básicas para funcionamento de UTIs. Nela inclui a assistência odontológica a beira leito, deixando subentendido que o CD faz parte da equipe multidisciplinar. Essa resolução entrou em vigor três anos após sua publicação, mas não especifica a carga horária mínima para este profissional ${ }^{19}$.

A portaria 1032/2010 inclui serviços odontológicos na tabela de procedimentos ofertados pelo SUS a pacientes com necessidades especiais tratados em hospitais. Além, de ressarcir aos profissionais que realizam esses procedimentos de alta complexidade, garante também que procedimentos antes feitos na atenção primária e secundária, poderão ser realizados na atenção terciária ${ }^{20}$. Porém, não há clareza no conceito do que é um paciente especial, o que pode gerar interpretações diversas, como exclusão de indivíduos que não possuem deficiências cognitivas e motoras.

Já a OH enquanto uma Habilitação Odontológica, foi regulamentada pelo Conselho Federal de Odontologia (CFO), através da resolução CFO-162/2015. As áreas de atuação do CD habilitado em $\mathrm{OH}$ incluem inserção deste profissional em ambiente hospitalar, prestação de assistência odontológica a paciente internado, home care e em serviços de urgência e emergência. Compreende ainda, atuar em suporte básico de vida, realizar pesquisas para melhora de diagnósticos, prevenção e tratamento além, de avaliação epidemiológica em ambiente hospitalar ${ }^{21}$.

Alguns estados e municípios brasileiros percebendo a necessidade da atuação desse profissional têm aprovados suas próprias leis. No estado do Paraná entrou em vigor a Lei 18.120/2014, que tornou obrigatória a prestação de assistência odontológica aos pacientes internados e em atendimento nos hospitais gerais, que contam com a capacidade normal de operação de mais de cinquenta leitos ${ }^{22}$. Já no Distrito Federal, entrou em vigor a Lei 5.744/2016, que dispõe sobre o direito constitucional à saúde bucal no SUS onde haja pacientes internados ${ }^{23}$.

O município de São Paulo aprovou em fevereiro de 2018 a Lei 16.860, que Institui a Política Municipal de Proteção à Saúde Bucal da Pessoa Hospitalizada ${ }^{24}$. Em abril de 2018, o estado de Alagoas deliberou a Lei 8.009 que coloca em prática a prestação de serviços odontológicos a pacientes crônicos de instituições hospitalares públicas e privadas ${ }^{25}$.

O estado do Mato Grosso do Sul adotou a Lei 5163/2018 que obriga a presença de profissionais de odontologia nas UTIs em hospitais públicos e privados ${ }^{26}$. Em São Luiz do Maranhão, a Lei Municipal 490/2019 tornou obrigatória a presença do CD na equipe multidisciplinar em todos os hospitais públicos e privados do município ${ }^{27}$.

O Conselho Estadual de Saúde de Minas, baseado nessas leis, criou a Resolução no 45/2018 que aprova as recomendações referentes à implementação de serviços odontológicos em ambiente hospitalar no Estado de Minas Gerais ${ }^{28}$. Solicitou que a Assembleia Legislativa de Minas Gerais realizasse a discussão e a elaboração de um projeto de lei que determinasse a inserção de profissionais da Odontologia habilitados nos hospitais públicos e privados, a nível estadual. A Câmara dos Deputados aprovou em abril de 2019, o Projeto de Lei 34/2013 (antigo PL 2776/2008), que obriga a presença de CD em hospitais públicos e privados ${ }^{29}$. Além de supervisionar outros profissionais habilitados, para atuar na área, fora das UTIs, o CD poderia realizar os atendimentos domiciliares, na modalidade home care. O Projeto de Lei foi vetado pelo Presidente da República do Brasil, em junho do mesmo ano. 
Devido à demora da aprovação de uma lei federal, a inserção de profissionais da área da odontologia em ambiente hospitalar acaba sendo limitada ${ }^{30}$. Alguns estados, ainda julgam desnecessária a presença do CD nos hospitais, deixando de contar um com profissional especializado, capaz de prevenir e tratar as afecções bucais. Essa condição determina o desemparo de uma parcela da população, que necessita do atendimento odontológico em condições diferenciadas.

\section{CONCLUSÃO}

A presença do CD no ambiente hospitalar proporciona o atendimento integral à saúde, podendo prevenir ou amenizar as complicações decorrentes do período de internação. Medidas como o reconhecimento da $\mathrm{OH}$ por parte dos Conselhos de Odontologia, a criação de leis que garantem o atendimento odontológico ao paciente hospitalizado e fiscalização do cumprimento das mesmas são um avanço na valorização e inserção do CD na atenção multidisciplinar ao paciente atendido nas Unidades de Terapia Intensiva, o que evidencia a importância do cuidado odontológico no ambiente hospitalar e proporciona um atendimento mais completo e integral ao paciente internado.

\section{REFERÊNCIAS}

1. Associação Brasileira de Odontologia Hospitalar (ABRAOH). Porto Alegre; 2016.

2. Godoi APT, Francesco AR, Duarte A. Odontologia hospitalar no Brasil: uma visão geral. Rev Odontol Unesp. 2009;38(2):105-9.

3. Eduardo FP, Bezinelli LM, Orsi E, Rodrigues M, Ribeiro MS, Hamerschlak N, et al. Influência dos cuidados odontológicos acompanhados de laserterapia sobre a mucosite oral durante transplante alogênico de células hematopoiéticas: estudo retrospectivo. Einstein (São Paulo) 2011;9(2):201-6.

4. Morais TMN, Silva A, Avi ALRO, Souza PHR, Knobel E, Camargo LFA. A importância da atuação odontológica em pacientes internados em unidade de terapia intensiva. Rev Bras Ter Intensiva. 2006;18(4):412-7.

5. São Paulo (Estado). Manual de odontologia hospitalar. São Paulo: Grupo Técnico de Odontologia Hospitalar; 2012.

6. Araújo RJG, Vinagre NL, Montoril ], Sampaio S. Avaliação sobre a participação de cirurgiões-dentistas em equipes de assistência ao paciente. Acta Sci Health Sci. 2009;31(2):153-7.

7. Araújo RG, de Oliveira LCG, Hanna LMO, Corrêa AM, Carvalho LHV, Álvares CF. Análise de percepções e ações de cuidados bucais. Rev Bras Ter Intensiva. 2009; 21(1):38-44.

8. Morais TMN. Fundamentos da odontologia em ambiente hospitalar/UTI. 1.ed. Rio de Janeiro: Elsevier; 2015

9. Silva OMP, Lebrão ML. A organização do atendimento da odontologia hospitalar e da traumatologia bucomaxilo-facial no município de São Paulo. Rev Odontol UNESP. 2001;30:43-54.

10. Paim JS. Reforma sanitária brasileira: contribuição para a compreensão e crítica. Rio de Janeiro: Fiocruz, 2008.

11. Brasil. Ministério da Saúde. Secretaria Nacional de Assistência à Saúde. ABC do SUS: doutrinas e princípios. Brasília; 1990

12. Dantas BO, Araujo IA, Araujo HBN, Araujo EC, Bezerra ACB, Miranda AF. Saúde bucal e cuidados na Unidade de Terapia Intensiva. Revista Odontol Planal Cent. 2015;5(1):28-32.

13. Comes SF, Esteves MCL. Atuação do cirurgião-dentista na UTI: um novo paradigma. Rev bras odontol. 2012;69(1):67-70.

14. Sannapieco FA. Relação entre doença periodontal e doenças respiratórias. In: Rose LF, Genco R], Cohen WD, Mealy B. Medicina periodontal. São Paulo: Santos; 2002. p. 83-97.

15. Diretrizes brasileiras para tratamento das pneumonias adquiridas no hospital e das associadas à ventilação mecânica - 2007. J bras pneumol. 2007;33 Suppl 1:51-30.

16. Souza AF, Guimaraes AC, Ferreira EF. Avaliação da implementação de novo protocolo de higiene bucal em um centro de terapia intensiva para prevenção de pneumonia associada à ventilação mecânica. Rev Mineira de enfermagem. 2013;17(1):177-84.

17. Rabelo GD, Queiroz CI, Santos PSS. Atendimento odontológico ao paciente em unidade de terapia intensiva. Arq Med Hosp Cienc Med Santa Casa São Paulo. 2010;55(2):67-70. 
18. Pereira RS, d'Ottaviano L. Saúde bucal dos pacientes internados na Unidade de Terapia Intensiva (UTI) Diretrizes normas e condutas. Serviço de Odontologia do HC, Campinas. [acesso 2019 nov 19]. Disponível em: http://www. fcm.unicamp.br/diretrizes/d_n_c/Saude_\%2obucal_\%2opacientes_uti/saude_bucal_pac_uti_pag_2.html.

19. Agência Nacional de Vigilância Sanitária (Brasil). Resolução nº. 7, de 24 de fevereiro de 2010. Dispõe sobre os requisitos mínimos para funcionamento de unidades de terapia intensiva e dá outras providências. Diário Oficial da União, 21 de agosto de 2006.

20. Brasil. Ministério da saúde. Portaria nº.1032, de 05 de maio de 2010. Inclui procedimento odontológico na tabela de procedimentos, medicamentos, órteses e próteses e materiais especiais do Sistema Único de Saúde - SUS, para atendimento às pessoas com necessidades especiais. Diário Oficial da União, Brasília, 5 de maio de 2010.

21. Conselho Federal de Odontologia. Resolução n ${ }^{\circ}$ CFO-162, de 09 de novembro de 2015. Conceitua a odontologia hospitalar e define a atuação do cirurgião-dentista habilitado a exercê-la. Diário Oficial da União, 6 de novembro de 2015.

22. Paraná. Lei $n^{\circ} 18120$, de 25 de junho de 2014 . Torna obrigatória a prestação de assistência odontológica aos pacientes em hospitais gerais. Diário oficial do Estado- PR, 2 de julho de 2014.

23. Distrito Federal. Lei $n^{\circ} 5.744$, de 9 de dezembro de 2016. Dispõe sobre o direito constitucional à saúde bucal no Sistema Único de Saúde do Distrito Federal onde haja internação de pacientes. Diário oficial da República Federativa do Brasil, Poder Executivo, 02 de julho de 2014.

24. São Paulo (Cidade). Lei nº 16.860, de 9 de fevereiro de 2018. Institui a Política Municipal de Proteção à Saúde Bucal da Pessoa Hospitalizada, e dá outras providências. Diário Oficial da Cidade, 10 de fevereiro de 2018, p. 3 C. 2-3.

25. Alagoas. Lei ordinária $n^{\circ} 8.009$, de 18 de abril de 2018. Dispõe a obrigatoriedade da prestação de assistência odontológica a paciente em regime de internação e portadores de doenças crônicas em unidades de saúde públicas e privadas do estado de Alagoas. Diário Oficial do Estado, - AL, 19 de abril de 2018.

26. Mato Grosso do Sul. Lei n 5.163, de 20 de março de 2018. Dispõe sobre a obrigatoriedade da presença de profissionais de Odontologia nas Unidades de Terapia Intensiva no Estado de Mato Grosso do Sul. Diário Oficial do Estado - MS, 20 de março de 2018, p.1.

27. São Luís. Lei Promulgada $n^{\circ} 490$, de 6 de março de 2018. Dispõe sobre a obrigatoriedade da presença do Cirurgião-Dentista na equipe multiprofissional dos hospitais do Município de São Luís, e dá outras providências. Diário Oficial do Município, São Luís, 12 de junho de 2018.

28. Minas Gerais. Resolução CESMG nº 045, de 10 de setembro de 2018. Dispõe sobre a aprovação de recomendações referente a implantação de Serviços de Odontologia Hospitalar no Estado de Minas Gerais. Diário Oficial do Estado - MD, 19 de dezembro de 2018, p. 70.

29. Brasil. Projeto de Lei da Câmara n 34, de 2013. Torna obrigatória a prestação de assistência odontológica a pacientes em regime de internação hospitalar, aos portadores de doenças crônicas e, ainda, aos atendidos em regime domiciliar na modalidade home care. [acesso 2019 nov 19]. Disponível em: https://www25.senado.leg. $\mathrm{br} /$ web/atividade/materias/materia/11297.

30. Puricelli E, Baeder F, Pucci I, Munaretto JC, Silveira JM. Hospitais: espaços para a atuação permanente da odontologia. Rev Assoc Paul Cir Dent. 2017;71 (1):14-20. 